\title{
Significance of Intra-abdominal Free Fluid Detected in Ultrasonography in the Clinical Assessment and Outcomes of Adult Patients Presenting to the Emergency Department Due to Abdominal Pain
}

Aylin Erkek ${ }^{1}$, Yasemin Yılmaz Aydın ${ }^{2}$, Handan Çiftçi ${ }^{3}$, Hayri Ramadan ${ }^{4}$, Kerim Temiz ${ }^{5}$, Kuzey Aydinuraz ${ }^{6}$, Figen Coskun ${ }^{7}$

1. Emergency Medicine, SBU Kocaeli Derince Training and Research Hospital, Kocaeli, TUR 2. Emergency Medicine, Dışkapı Yıldırım Beyazıt Training and Research Hospital, Ankara, TUR 3. Emergency Medicine, Kars Kafkas University Faculty of Medicine, Kars, TUR 4. Emergency Medicine, Ankara Education and Research Hospital, Ankara, TUR 5. Radiology, Samsun Vezirköprü State Hospital, Samsun, TUR 6. General Surgery, Kırıkkale University Faculty of Medicine, Kırıkkale, TUR 7. Emergency Medicine, Dokuz Eylül University Faculty of Medicine, İzmir, TUR

$\square$ Corresponding author: Aylin Erkek, aylinerkek@hotmail.com

\section{Abstract}

\section{Objective}

The aim of the study was to evaluate the diagnostic process and clinical course in adult patients who presented to the emergency department (ED) with acute abdominal pain (AAP) and were found to have intra-abdominal free fluid (FF) on ultrasonography (USG).

\section{Methods}

This prospective observational study was conducted in a training and research hospital adult emergency department between March 15, 2013, and April 15, 2013. The study included 252 patients aged above 18 years, who were admitted to the emergency room complaining of nontraumatic acute abdominal pain and provided consent for the study.

\section{Results}

The most common diagnoses were acute, nonspecific abdominal pain (37.3\%), acute appendicitis (19\%), and urinary tract pathology (15.9\%). Intra-abdominal free fluid was detected with ultrasonography in $42.5 \%$ of patients. Patients with intra-abdominal free fluid were younger than the other patients. The emergency department length of stay was longer in patients with intra-abdominal free fluid ( $\mathrm{p}=0.011)$. Of the 252 patients enrolled in the study, $32.9 \%$ were admitted to the hospital, $21.4 \%$ of whom underwent surgery and $11.5 \%$ received medical therapy. Most of the patients (64.5\%) who were discharged home had no intraabdominal free fluid in the ultrasonography $(\mathrm{p}<0.001)$.

\section{Conclusion}

The presence of intra-abdominal free fluid alone did not guide the clinical decision regarding the diagnostic evaluation of adult patients that presented to the emergency department complaining of non-traumatic acute abdominal pain.

\section{How to cite this article}

Erkek A, Yılmaz Aydın Y, Çiftçi H, et al. (October 21, 2019) Significance of Intra-abdominal Free Fluid Detected in Ultrasonography in the Clinical Assessment and Outcomes of Adult Patients Presenting to the Emergency Department Due to Abdominal Pain. Cureus 11(10): e5948. DOI 10.7759/cureus.5948 
Categories: Emergency Medicine, Radiology, General Surgery

Keywords: acute abdominal pain, intraabdominal free fluid, ultrasonography

\section{Introduction}

Acute abdominal pain (AAP) is one of the most common causes of emergency department (ED) visits, accounting for approximately 5\%-10\% [1-2]. Medical history, physical examination, and laboratory and radiologic workup may not prove diagnostic at the initial assessment so followup is frequent, and only after this are $20 \%$ admitted to the hospital wards [2-3]. Although intraabdominal free fluid (FF) can be the main finding of acute inflammation in non-traumatic AAP, it can also be detected in subjects without any physiological complaint [2-3]. The aim of the present study was to evaluate the diagnostic process and clinical course in adult patients who presented to the ED complaining of AAP and who were found to have intra-abdominal FF on ultrasonography.

\section{Materials And Methods}

This study was conducted in a training and research hospital in Turkey that has approximately 220,000 ED admissions per year. Patients with AAP that were admitted to the ED between March 15, 2013, and April 15, 2013, were evaluated. Patients aged above 18 years, with nontraumatic AAP and who provided consent for the study, enrolled. The exclusion criteria were pregnancy, known intra-abdominal malignancy, congestive heart failure, and undergoing an abdominal operation within the last month.

On-call physicians in the ED performed the clinical assessment. Age, gender, presence of a known disease, past intra-abdominal operation, drug use, menstrual cycle; characteristics, duration, location, and extension of abdominal pain; the presence of accompanying complaints, and conditions that aggravate or attenuate symptoms were documented. Tenderness on palpation, location of tenderness, guarding, and rebound tenderness were all recorded. A complete blood count was obtained in all patients. Basic metabolic profile and urine tests were obtained for the differential diagnosis.

Ultrasonography (USG) was performed by the same radiologist in all patients upon admission. The presence, amount, and location of intra-abdominal FF and pathological findings were recorded (Toshiba Xario USTS-770A, Tokyo, Japan; convex 3.5 MHz and flat 7.5 MHz probes).

ED length of stay, consultations, discharge and hospitalization status, medical or surgical treatments after hospitalization, and final diagnoses were recorded. Three days after discharge, the patients were called to check whether the pain persisted or they were admitted to or hospitalized in another health center.

The control group comprised 38 adult subjects among the hospital staff, without any abdominal pain, any other acute complaint, pregnancy, congestive heart failure, known intra-abdominal malignancy, or abdominal operation within the last month, and who provided consent for the study. They all underwent an ultrasonographical assessment by the same radiologist.

The statistical analysis was performed using the IBM SPSS for Windows version 21.0 software package (IBM Corp., Armonk, NY, US). Numeric variables were expressed as mean \pm standard deviation and median [minimum - maximum] values. Categorical variables were expressed as number and percentage. The presence of a difference in categorical variables between the groups was investigated using the chi-square test and Fisher's exact test. The normality of numeric variables was assessed using the Shapiro-Wilk test, and the homogeneity of variances was assessed using the Levene test. The differences in numeric variables between the two independent groups were tested using the t-test if parametric test assumptions were met. The 


\section{Cureus}

Mann-Whitney U-test was used if parametric test assumptions were not met. The KruskalWallis test was used to compare more than two independent groups. The level of significance was $\mathrm{p}<0.05$.

The hospital ethics committee issued approval for the study protocol dated 06.03.2013, numbered 497, and with decision number 4110. The study was performed in accordance with the ethical standards laid down in the 1964 Declaration of Helsinki.

\section{Results}

In 2013, 20,244 patients were admitted to the ED between March 15 and April 15; 1535 (7.6\%) of whom were complaining of abdominal pain. The study included 275 patients with AAP who met the inclusion criteria and provided consent for participation in the study. Thirteen patients were lost to follow-up. Ten patients who were assessed ultrasonographically by another radiologist were excluded. The final study population consisted of 252 patients. The control group consisted of 38 healthy adults.

\section{Characteristics of the study group}

One hundred and fifty-nine (63.1\%) were females. The mean age was $39.4 \pm 17.5$ years (min-max: 18-87). Seventy-three percent of the patients did not have a known chronic disease while $18.7 \%$ had one, $6.7 \%$ had two, and $1.6 \%$ had at least three diseases. Seventy-one (28.2\%) had undergone one to four prior abdominal surgeries.

\section{Characteristics of the study group related to abdominal pain}

The onset of pain was one to four hours in 51 (20.2\%), and 25-48 hours in 120 patients (47.6\%). Of the patients, 159 (63.1\%) reported pain in one quadrant and 23 (9.1\%) reported widespread pain in four quadrants. The right lower quadrant was the most common site of pain (123 patients, 48.2\%) (Table 1).

Feature

\section{Value}

n

$\%$

Duration of pain (hour)

$1-4$

$5-24$

21

8.3

$25-48$

120

47.6

$49-120$

60

23.8

Pain location upon admission

Right lower quadrant

Right upper quadrant

Left lower quadrant

52

20.6

Umbilical

Epigastric

23
9.1 


\section{Cureus}

$\begin{array}{ll}\text { Widespread } & 23\end{array}$

$\begin{array}{lr}\text { Left upper quadrant } & 16\end{array}$

Suprapubic

Course of pain

$\begin{array}{ll}\text { No change } & 124\end{array}$

Gradual Increase

Gradual Decrease

Colic

Presence of factors aggravating pain

Movement

Eating

Coughing

Lying on the back

Presence of alleviating factors

Lying on the back

Vomiting 4

Eating

Presence of complaints accompanying pain

Nausea

Vomiting

Dysuria

Pollakiuria

29

Constipation

16

Diarrhea 15

Fever

9

TABLE 1: Pain characteristics of patients that were evaluated for acute abdominal pain 


\section{Cureus}

The abdomen was tender to palpation in a single quadrant in 152 patients $(61.5 \%)$, and there was widespread tenderness in four quadrants in 17 patients $(6.7 \%)$ (Table 2$)$.

\section{Feature}

\section{Value}

$\mathrm{n}$

$\%$

Appearance of the abdomen

Presence of scar 66

Presence of distension

2

0.8

Bowel sounds

Normal

Decreased

Increased

Tenderness to palpation

Single area

2 areas

3 areas

Location of tenderness tenderness

Right lower quadrant

Right upper quadrant

Umbilical area

Mc-Burney's point

Suprapubic area

Murphy's point 16

Left upper quadrant

Epigastric area

14

Costovertebral angle tenderness

15

6

Presence of Guarding

52

20.6

Presence of Rebound Tenderness

97 


\section{Cureus}

\section{TABLE 2: Physical examination findings on admission in patients that were evaluated}

for acute abdominal pain

\section{Laboratory and imaging findings of the study group}

A hemogram was obtained from all patients at the time of admission, from 55 patients (21.8\%) at four hours of the follow-up period, and from three patients (0.12\%) at eight hours of the follow-up period. All patients underwent an abdominal USG. The characteristics and diagnoses are given in Table 3 and Table 4.

\section{Feature}

White blood cell count $/ \mathrm{mm} 3$

On admission

At 4 hours $(n=55)$

At 8 hours $(n=3)$

Haematocrit

On admission

At 4 hours $(n=55)$

At 8 hours $(n=3)$

Presence of leukocytosis $(>11000 / \mathrm{mm} 3)$

On admission

At 4 hours

At 8 hours

Elevated alanine aminotransferase level (>100 IU)

On admission

At 4 hours

Elevated aspartate aminotransferase level (>100 IU)

On admission

At 4 hours

Elevated amylase Level (>200 IU)

On admission

At 4 hours
Value

Mean \pm SD

Min.-Max

$11233 \pm 4236$

$4460-31000$

$11500 \pm 4025$

5100-22800

$11066 \pm 2610$

$8600-13800$

$41.7 \pm 5.2$

15.9-57.3

$39.5 \pm 5.2$

28.9-54

$41.2 \pm 1.3$

$40.4-42.8$

$\mathrm{n}$

$\%$

105

41.7

27

1

0.4

6

2
2 


\section{Cureus}

Urine analysis

Erythrocyturia

Leukocyturia

Nitrite positivity

Ketone positivity
170

67.5

50

19.8

70

27.8

7
0.4

2.8

TABLE 3: Laboratory findings of patients that were evaluated for acute abdominal pain 


\section{Cureus}

Feature

Feature

Free fluid in the USG (+)

Quantity of free fluid in the USG

Mild

Minimal $(<2 \mathrm{~cm})$

Moderate $(2-5 \mathrm{~cm})$

Severe $(>5 \mathrm{~cm})$

Localization of free fluid in the USG

Between bowel loops

Douglas pouch

Pelvic region

Pericholecystic area

Pararenal area

Paraovarian area

Perivesical area

Peripancreatic area

Between bowel loops + Douglas pouch

Between bowel loops + Pericholecystic area

Between bowel loops + Gastrohepatic

1

Between bowel loops + Paraovarian area

Between bowel loops + Douglas pouch + Paraovarian area

TABLE 4: Characteristics of free fluid in the ultrasonography assessment of patients in the study group

USG: Ultrasonography

\section{Findings related to follow-up and treatment in the study group}

Nonspecific abdominal pain was most common (94 patients, 37.3\%), followed by acute appendicitis (48 patients, 19\%), urinary tract pathology (40 patients, $15.9 \%$ ), gynecologic disorders (22 patients, 8.7\%), gall bladder diseases (18 patients, 7.1\%), bowel pathologies requiring surgery ( 9 patients, $3.6 \%$ ), acute pancreatitis (6 patients, $2.4 \%$ ), ischaemic pathologies 


\section{Cureus}

(2 patients, $0.8 \%$ ), abdominal mass and abscess ( 2 patients, $0.8 \%$ ), diverticulitis ( 2 patients, $0.8 \%$ ), and other abdominal pathologies (9 patients, $3.6 \%$ ). The definitive diagnosis after surgery was acute appendicitis in 43 patients (17.1\%). Other data are given in Table 5.

\section{Feature}

1-2 hours

3-4 hours

5-8 hours

$>8$ hours

Consultation (+)

Requested Consultation

General Surgery

Gynecology

Other Branches

Outcomes in the emergency room

Discharged from the emergency room

Hospitalized

Left with their own wish

Treatment of hospitalized patients

Surgery

Medical therapy
Emergency Room Length of Stay

\section{Value}

$\%$

26 156

60

10

4

165

54

\section{2}

TABLE 5: Emergency room follow-up and assessment data of patients in the study group
On Day 3 after discharge, all 169 patients were called to obtain information about their clinical condition. Forty-eight patients (19\%) were admitted to another healthcare facility due to abdominal pain. Twenty-six (10.3\%) received medical therapy, whereas two patients $(0.8 \%)$ underwent surgery (one cholecystitis and one appendicitis). Fourteen patients (5.6\%) continued outpatient control visits and two patients $(0.8 \%)$ were hospitalized in another center for medical therapy. Of these patients, $4.4 \%(n=11)$ had not been diagnosed yet.

\section{Comparison and analysis of the data of the study group}


Patients with intra-abdominal FF in USG were younger while the FF incidence was lower in postmenopausal women $(\mathrm{p}<0.000, \mathrm{p}<0.000)$. In female patients, FF was located between the bowel loops and pelvis ( $\mathrm{p}<0.000)$.

The majority of patients with findings suggestive of a non-surgical or infectious condition did not have FF ( $\mathrm{p}=0.03)$. FF was more common in patients with an aggravating factor and without an alleviating factor ( $\mathrm{p}=0.031, \mathrm{p}=0.038$ ). FF accompanied by a pathological diagnosis was significant for older patients ( $\mathrm{p}=0.002$ ). The presence and location of $\mathrm{FF}$, as well as abdominal tenderness, were more significant in patients with right upper quadrant pain $(\mathrm{p}<0.00, \mathrm{p}=0.006$, $\mathrm{p}=0.015)$. FF was accompanied by guarding $(\mathrm{p}=0.019)$ and rebound tenderness $(\mathrm{p}=0.041)$. FF was associated with both air-fluid levels on plain abdominal X-ray and the presence of a pathological diagnosis $(\mathrm{p}=0.035, \mathrm{p}=0.02)$.

There was no FF in the majority of patients with nonspecific abdominal pain and patients who were discharged home while there was FF in the majority of patients diagnosed with a gynecologic disorder $(\mathrm{p}<0.01, \mathrm{p}<0.00, \mathrm{p}<0.001)$. FF alone was more common in patients who received medical therapy $(\mathrm{p}=0.02)$.

The majority of patients who stayed one to two hours in the ED had no FF ( $p=0.011$ ). Consultation requests and hospitalization were more frequent in patients with $\mathrm{FF}$ and ultrasonographical pathological diagnosis than the detection of FF only $(p=0.008, p<0,001)$.

Ultrasonographical pathology appeared to be the major factor leading to the decision to perform surgery ( $\mathrm{p}=0.033$ ). Twenty-two patients with FF and a pathological diagnosis (55\%), six patients with FF but without a pathological diagnosis (66.7\%), and 21 patients without FF but with a pathological diagnosis (84\%) underwent surgery. Patients with ultrasonographical detection of both FF and pathologic diagnosis had significant re-admission rates $(\mathrm{p}<0.001)$ (Table 6). 


\section{Cureus}

\begin{tabular}{|c|c|c|c|c|c|}
\hline \multirow{3}{*}{$\begin{array}{l}\text { Feature } \\
\text { Consultation request }\end{array}$} & \multicolumn{5}{|c|}{ Presence of Free fluid } \\
\hline & \multicolumn{2}{|c|}{ positive } & \multicolumn{2}{|c|}{ negative } & \multirow[b]{2}{*}{$\mathrm{p}$} \\
\hline & $\mathrm{n}$ & $\%$ & $\mathrm{n}$ & $\%$ & \\
\hline Requested & 96 & 58.2 & 69 & 41.8 & \multirow{2}{*}{$0.00 *$} \\
\hline Not requested & 11 & 12.6 & 16 & $8 / .4$ & \\
\hline \multicolumn{6}{|l|}{ Hospitalization decision } \\
\hline Discharged & 56 & 34.6 & 106 & 65.4 & \multirow{2}{*}{$0.00^{*}$} \\
\hline Hospitalization & 49 & 59 & 34 & 41 & \\
\hline \multicolumn{6}{|l|}{ Inpatient treatment } \\
\hline Operated & 27 & 50 & 27 & 50 & \multirow{2}{*}{$0.02^{*}$} \\
\hline Medical therapy & 22 & 75.9 & 7 & 24.1 & \\
\hline \multicolumn{6}{|c|}{ Admission to another healthcare facility } \\
\hline Yes & 17 & 35.4 & 31 & 64.6 & \multirow{2}{*}{0.992} \\
\hline No & 41 & 33.9 & 80 & 66.1 & \\
\hline
\end{tabular}

TABLE 6: Presence or absence of free fluid in treatment decisions

\section{Characteristics of the control group}

Of the 38 patients in the control group, 21 (55.3\%) were females. The mean age was $29.1 \pm 10.28$ years (min-max=18-56). There was no FF in 36 subjects $(94.7 \%)$ in the control group, a finding different from the patient group ( $\mathrm{p}<0.000)$. Of the two patients with FF, only one had ultrasonographically detected pathology, which was an ovarian cyst.

\section{Discussion}

AAP is one of the most common causes of ED visits. Intra-abdominal FF is one of the findings of acute inflammation in non-traumatic AAP but it can also be detected in patients without any complaints $[1-2,4]$. The demographic properties of the study population were consistent with studies by Hastings et al. and Cooper et al., reporting female gender predominance and similar figures for incidence, mean age of AAP admissions, as well as pain distribution [5-6]. Guarding was present in $20 \%$ and rebound tenderness in $38 \%$ of patients, with $57.4 \%$ guarding and $92.6 \%$ rebound tenderness present in patients who underwent surgery in accordance with the literature [7-8]. The majority of patients requiring emergency surgery or hospitalization had leucocytosis, similar to the study by Çalışkan et al [8]. In a meta-analysis by Andersson et al., the positive likelihood ratio was 2.47 for the diagnosis of acute appendicitis [9]. Follow-up was one to four hours in $72.2 \%$ of the patients, similar to $65.9 \%$ in a study by Aygencel et al. In the present study, $32.9 \%$ of patients were hospitalized, $21.4 \%$ of which required surgery consistent with the literature [3]. Different rates of hospitalization are reported in studies by Hastings et al. and Lindelius et al. [4,10]. 
USG is an important imaging method for AAP due to its low cost, easy accessibility, lack of radiation exposure, and contrast agent with the rate of accurate diagnosis increasing from $70 \%$ to $83 \%$ when USG is performed after clinical assessment [11].

In the present study, USG showed normal findings in $59.5 \%$ of the patients, which is higher than the incidence of normal ultrasonographical findings in other studies [12]. Acute appendicitis (14.3\%), gynecologic problems (8.7\%), and gall bladder problems (7.1\%) were the most frequent diagnoses in our study.

Intra-abdominal FF observed on imaging could also be physiologic. FF frequency was 30\%-40\% in healthy female volunteers of reproductive age [13-14]. Brown and Dubbins reported intraabdominal FF in $40 \%$ of healthy voluntary males [15]. In the present study, USG showed FF in two out of 38 subjects in the control group (5.3\%).

Patients with FF were significantly younger in our study while patients with pathological findings and FF were older. Rathaus et al. evaluated intra-abdominal FF in 266 patients with acute and/or chronic abdominal pain versus 396 asymptomatic pediatric patients and reported a significantly higher rate of FF in children with abdominal pain (28.6\%) as compared to asymptomatic children (7\%). Gender did not affect the presence of FF in the present study, similar to results by Rathaus et al. [16]. Patients that had FF in the Douglas and pelvic areas were also significantly younger while $85.4 \%$ of postmenopausal women did not have FF. The presence of FF was not affected by gynecologic factors in our study despite studies reporting an increase in physiologic FF in asymptomatic adult females in the ovulatory period [17].

It can be argued that the absence of guarding and rebound tenderness may be suggestive of the absence of FF, as USG failed to show FF in the majority of patients showing no guarding and rebound tenderness.

FF was mostly detected within the bowel loops (63.6\%), Douglas pouch (9.3\%), and pelvic area (5.6\%). The predominance of fluid accumulation within the bowel loops is consistent with the results of a study by Jequier et al. in which FF was mostly located within the bowel loops (68\%) again [18].

In our study, $65 \%$ of patients without any pathological finding did not have FF. The majority of patients with normal USG or findings suggestive of a non-surgical or infectious condition did not have FF while FF was common in patients with a gynecologic or urologic condition. Similarly, in the study by Matz et al., the final diagnosis was of gynecologic or urologic origin in $36 \%$ of patients who had intra-abdominal FF alone [19].

In our study, there was a difference in consultation requests depending on the presence of FF and ultrasonographical pathological findings. The presence of FF did not indicate a requirement for consultation; however, $87.4 \%$ of patients for which consultation was not requested did not have FF. On the other hand, a consultation was mostly requested in the presence of FF accompanied by ultrasonographically diagnosed pathology (98.2\%). In the present study, the absence of FF affected the ED length of stay. Seventy-six point nine percent (76.9\%) of patients with one to two hours of ED had no intra-abdominal FF.

USG did not show FF in $64.5 \%$ of discharged patients. The presence of FF did not favor surgical therapy versus medical therapy and $75.9 \%$ of patients with FF alone received medical therapy after hospitalization.

The hospitalization rate was $74.1 \%$ for patients with both FF and pathological conditions while it was $17.6 \%$ for patients with FF alone. Matz et al. found a discharge rate of $48.8 \%$ for patients 
with FF but without a confirmed surgical pathology [19]. Also, the presence of FF alone did not affect re-admission to another health care facility $(\mathrm{p}=0.99)$.

In our study, FF was present in $42.5 \%$ of 252 patients who presented to the ED due to nontraumatic acute abdominal pain. The clinical significance of intra-abdominal FF was investigated in 407 pediatric patients with AAP in the study by Matz et al. where FF could be visualized in $33 \%$ of the patients. They investigated the importance of intra-abdominal FF in leading to the decision of surgery and found that intra-abdominal FF alone did not influence the decision of surgery in the absence of a pathological finding on imaging studies [19]. In their study group, $40.3 \%$ of patients with intra-abdominal FF plus confirmed surgical pathology underwent surgery while only $6.3 \%$ of children with intra-abdominal FF alone underwent surgery [19].

\section{Limitations}

The present study may have limitations due to the fact that only patients admitted to the ED of one hospital and patients who were ultrasonographically assessed by a single radiologist were included in the study.

\section{Conclusions}

The present study is an original study that evaluated FF using USG in adult patients with nontraumatic acute abdominal pain. Ultrasonographical FF did not necessarily indicate an intraabdominal pathology and did not favor surgical therapy as compared to medical therapy. Of the patients with FF but without a detectable pathology, 82\% were discharged from the ED. The presence of intra-abdominal FF alone was not found to guide the assessment of adult patients with non-traumatic abdominal pain.

\section{Additional Information}

\section{Disclosures}

Human subjects: Consent was obtained by all participants in this study. Ethics Committee of Ankara Education and Research Hospital, Ankara, issued approval 497/4110. The hospital ethics committee issued an approval for the study protocol dated 06.03.2013 and numbered 497 and with decision number 4110. Animal subjects: All authors have confirmed that this study did not involve animal subjects or tissue. Conflicts of interest: In compliance with the ICMJE uniform disclosure form, all authors declare the following: Payment/services info: All authors have declared that no financial support was received from any organization for the submitted work. Financial relationships: All authors have declared that they have no financial relationships at present or within the previous three years with any organizations that might have an interest in the submitted work. Other relationships: All authors have declared that there are no other relationships or activities that could appear to have influenced the submitted work.

\section{References}

1. Kamin RA, Nowicki TA, Courtney DS, Powers RD: Pearls and pitfalls in the emergency department evaluation of abdominal pain. Emerg Med Clin North Am. 2003, 21:61-72. 10.1016/S0733-8627(02)00080-9

2. McNamara R, Dean AJ: Approach to acute abdominal pain. Emerg Med Clin North Am. 2011, 29:159-173.

3. Graff LG IV, Robinson D: Abdominal pain and emergency department evaluation. Emerg Med Clin North Am. 2001, 19:123-136.

4. Hastings RS, Powers RD: Abdominal pain in the ED: a 35 year retrospective . Am J Emerg Med. 
2011, 29:711-716. 10.1016/j.ajem.2010.01.045

5. Cooper JG, Hammond-Jones D, O'Neill E, et al.: The clinical decision unit has a role to play in the management of acute undifferentiated abdominal pain. Eur J Emerg Med. 2012, 19:323328. 10.1097/MEJ.0b013e32834da85d

6. Pappas A, Toutouni H, Gourgiotis S, et al.: Comparative approach to non-traumatic acute abdominal pain between elderly and non-elderly in the emergency department: a study in rural Greece. J Clin Med Res. 2013, 5:300-304. 10.4021/jocmr1424W

7. Gerhardt RT, Nelson BK, Keenan S, Kernan L, MacKersie A, Lane MS: Derivation of a clinical guideline for the assessment of nonspecific abdominal pain: the Guideline for Abdominal Pain in the ED Setting (GAPEDS) Phase 1 Study. Am J Emerg Med. 2005, 23:709-717.

8. Caliskan M, Coskun A, Acar A, et al.: Multivariate prospective evaluation of patients admitted with acute abdominal pain in emergency surgery clinics. Eurasian J Emerg Med. 2010, 9:75-82. 10.4170/jaem.2010.14238

9. Andersson REB: Meta-analysis of the clinical and laboratory diagnosis of appendicitis . Br J Surg. 2004, 91:28-37. 10.1002/bjs.4464

10. Lindelius A, Törngren S, Pettersson H, Adami J: Role of surgeon-performed ultrasound on further management of patients with acute abdominal pain: a randomised controlled clinical trial. Emerg Med J. 2009, 26:561-566.

11. Allemann F, Cassina $P$, Röthlin M, Largiadèr F: Ultrasound scans done by surgeons for patients with acute abdominal pain: a prospective study. Eur J Surg. 1999, 165:966-970.

12. Yeniocak S, Turkmen S, Uzun O, et al.: Analysis of patients presenting to emergency department with acute abdominal pain. Eurasian J Emerg Med. 2012, 11:212-215.

13. Sirlin CB, Casola G, Brown MA, Patel N, Bendavid EJ, Deutsch R, Hoyt DB: US of blunt abdominal trauma: importance of free pelvic fluid in women of reproductive age. Radiology. 2001, 219:229-235. 10.1148/radiology.219.1.r01ap45229

14. Davis JA, Gosink BB: Fluid in the female pelvis: cyclic patterns . J Ultrasound Med. 1986, 5:7579. 10.7863/jum.1986.5.2.75

15. Brown SE, Dubbins PA: Detection of free intraperitoneal fluid in healthy young men . J Ultrasound Med. 2012, 31:1527-1530. 10.7863/jum.2012.31.10.1527

16. Rathaus V, Grunebaum M, Konen O, Odsatchy A, Zissin R, Shapiro M, Werner M: Minimal pelvic fluid in asymptomatic children: the value of the sonographic finding. J Ultrasound Med. 2003, 22:13-17. 10.7863/jum.2003.22.1.13

17. Ormsby EL, Geng J, McGahan JP, Richards JR: Pelvic free fluid: clinical importance for reproductive age women with blunt abdominal trauma. Ultrasound Obstet Gynecol. 2005, 26:271-8. 10.1002/uog.1981

18. Jéquier S, Jéquier JC, Hanquinet S: Intraperitoneal fluid in children: normal ultrasound findings depend on which scan head you use. Pediatr Radiol. 2003, 33:86-91. 10.1007/s00247002-0837-X

19. Matz S, Connell M, Sinha M, Goettl CS, Patel PC, Drachman D: Clinical outcomes of pediatric patients with acute abdominal pain and incidental findings of free intraperitoneal fluid on diagnostic imaging. J Ultrasound Med. 2013, 32:1547-53. 